\title{
SISTEMAS SILVOPASTORILES EN BOSQUES DE Nothofagus antarctica REVISIÓN DEL CONOCIMIENTO ACTUAL EN PATAGONIA SUR, ARGENTINA
}

Peri, Pablo ${ }^{1}$

\section{RESUMEN}

Los sistemas silvopastoriles, que combinan en una misma unidad de superficie árboles con pasturas o pastizales bajo pastoreo con ganado, son una alternativa productiva en la región Patagónica, y podrían constituir una actividad ecológica, social y económicamente viable.

El objetivo del presente trabajo es presentar la información generada en Patagonia sobre los sistemas silvopastoriles en bosques nativos de ñire (Nothofagus antarctica) con pastizales naturales y, en menor medida, con introducción de pasturas.

Se presenta información productiva respecto a sus tres principales componentes; silvicultura y producción del componente arbóreo, producción forrajera del sotobosque y producción y manejo animal. Además, se resaltan los avances en los estudios de ecofisiología (nutrientes, fijación de carbono) y pautas para la conservación del ñirantal bajo uso silvopastoril.

Se ha avanzado en el conocimiento de estos sistemas, principalmente en la producción y calidad del componente forrajero e interacciones con el estrato arbóreo. Sin embargo, resta profundizar aspectos relacionados al manejo animal a escala de establecimiento durante todo un ciclo productivo, fortalecer la factibilidad de instalación de industrias primarias o secundarias alternativas para aumentar el valor agregado de los productos madereros y, en el caso de los sistemas silvopastoriles en bosque nativo, son necesarios futuros estudios que tengan en cuenta el impacto a nivel paisaje y la conectividad para la vida silvestre a escala regional.

Palabras claves: Ganadería, ñire, pastizal, silvicultura, Patagonia

1 EEA INTA Santa Cruz - UNPA - CONICET. Casilla de Correo 332, (CP 9400), Río Gallegos, Santa Cruz, Argentina, email: pperi@correo.inta.gov.ar 


\section{SILVOPASTORAL SYSTEMS IN Nothofagus antarctica FORESTREVIEW OF THE ACTUAL KNOWLEDGE IN SOUTHERN PATAGONIA, ARGENTINA}

\section{SUMMARY}

Silvopastoral systems, that combine trees and grasslands or pastures under grazing in the same unit of land, would be an economical, ecological and social productive alternative in Patagonia.

Therefore, the aim of this work is to review the available information related to silvopastoral systems in Patagonia in Nothofagus antarctica native forest mainly with natural understorey as a forage resource and in less extent with introduced pastures. Information about silviculture and timber production, understorey forage production and livestock management and production are provided.

Also, other aspects of silvopastoral systems such as ecophysiology studies (nutrients and carbon fixation) and conservation information are outlined in this work.

There are advances in knowledge related mainly to quality and production of the understorey component interacting with tree strata. However, research on sheep and cattle production at farm level over a year, the improve of primary and secondary timber industry that incorporate value to the harvested wood, and further studies that incorporate the impact on landscape and fauna connectivity at regional level, are needed. Integrated economical information and social aspects of silvopastoral systems in the region also are important to be developed.

Key Words: Grassland, livestock, ñire, silviculture, Patagonia 


\section{INTRODUCCION}

Patagonia Argentina ( $37^{\circ}$ a $55^{\circ}$ LS) es una amplia región (197 millones de hectáreas) que presenta una gran diversidad de climas y ambientes, destacándose la estepa (representando $\sim 93 \%$ de la superficie total), el ecotono $(\sim 3,7 \%)$, los valles $(\sim 1,5$ $\%$ ) y bosques nativos ( 1,8\%). Como característica principal presenta un gradiente de precipitación que decrece de oeste a este, de 4000 a $200 \mathrm{~mm}$ anuales.

La producción ganadera (principalmente ovina) se extendió en Patagonia Sur (Chubut, Santa Cruz y Tierra del Fuego) a fines del siglo XIX, con un aumento paulatino en el número de animales a través del tiempo (según el Instituto Nacional de Estadística y Censos-INDEC, en el año 2002 existían 6.517 .580 ovinos, 211.575 bovinos y 86.850 equinos) lo que determinó la presencia de la ganadería en la zona de la cordillera.

Los sistemas silvopastoriles que combinan en una misma unidad de superficie árboles con pasturas o pastizales nativos bajo pastoreo con ganado, son una alternativa productiva en Patagonia y podrían constituir una actividad ecológica, social y económicamente viable. Estos sistemas deben ser diseñados de manera tal que se favorezcan las interacciones ecológicas beneficiosas que se manifiestan en un incremento de la producción, en la eficiencia del uso de los recursos y también en aspectos del medio ambiente.

El ñire es una especie nativa de los bosques patagónicos con una distribución amplia que se extiende desde el norte de Neuquén hasta Tierra del Fuego ocupando una superficie de 751.643 ha (SAyDS, 2005), de la cual aproximadamente el $70 \%$ tiene un uso silvopastoril. En particular, en Santa Cruz y Tierra del Fuego existen 97 estancias con bosque de ñire de las cuales un $68 \%$ tiene más del $10 \%$ de su superficie ocupada con bosque de ñire (Ormaechea et al., 2009).

La importancia de los bosques nativos de ñire como sistemas silvopastoriles principalmente radica en la capacidad productiva ganadera (ovina y bovina) y la obtención de productos maderos provenientes de las intervenciones silvícolas como postes, varas y leña.

El objetivo del presente trabajo es presentar la información generada en Patagonia Argentina sobre los sistemas silvopastoriles en bosques nativos de Nothofagus antarctica. 


\section{SILVICULTURA Y PRODUCCIÓN DEL COMPONENTE ARBÓREO}

Actualmente la propuesta silvícola en sistemas silvopastoriles con ñire contempla la intensidad de los raleos y aspectos relacionados a la continuidad del estrato arbóreo.

Integrando el conocimiento generado (Peri, 2005; Peri et al., 2005a,b; Sarasola et al., 2008a,b) y conceptos de practicidad operativa se proponen dos intensidades de raleo para diferentes sitios de ñirantales.

Mientras que en sitios de estrés hídrico severo (alturas de los árboles dominantes inferiores a los 5-8 m) se recomienda una intensidad máxima de raleo que deje una cobertura de copas remanente entre 50 y $60 \%$, en sitios con un régimen de precipitaciones más favorable (ñirantales con alturas de los árboles dominantes superiores a los $8 \mathrm{~m}$ ) se recomienda una intensidad máxima de raleo que deje una cobertura de copas remanente entre 30 y $40 \%$ (Peri et al., 2009a).

Sarasola et al. (2008b) evaluaron que la respuesta del crecimiento medio en diámetro de árboles de ñire al raleo fluctuó desde 0,18 a 0,49 cm/año para rodales densos y semiabierto, respectivamente.

Recientemente se generó un índice de densidad de rodal de ñirantales, independiente de la edad del rodal y la calidad de sitio, como una herramienta biométrica para determinar intensidades de raleo de modo de alcanzar diferentes coberturas arbóreas bajo un uso silvopastoril (Ivancich et al., 2009).

Ivancich et al. (2010), utilizando un modelo de crecimiento de rodal, estimó que el crecimiento volumétrico de los bosques de ñire fluctúa entre 1,3 a 8,3 $\mathrm{m}^{3} / \mathrm{ha} / \mathrm{año}$. Quedan excluidos de intervención silvícola aquellos bosques con alturas finales de árboles dominantes menores a los $4 \mathrm{~m}$ debido a la fragilidad ambiental del ecosistema (Quinteros et al., 2008).

Los volúmenes totales aprovechados en bosques de ñire bajo uso silvopastoril dependen de la calidad del sitio y la intensidad de los raleos. Por ejemplo, en la provincia de Santa Cruz, el volumen bruto con corteza aprovechado fluctuó desde $64 \mathrm{~m}^{3} / \mathrm{ha}$ para rodales con un remanente de transmisibilidad luminosa luego del raleo de $\sim 60 \%$ desarrollándose en Clase de Sitio (CS) V (altura media de árboles dominantes de 5,5 m) a $220 \mathrm{~m}^{3} / \mathrm{ha}$ en aquellos rodales creciendo en CS I (árboles dominantes de una altura media final mayor a $12 \mathrm{~m}$ ) y con una transmisibilidad luminosa remanente del $~ 30 \%$. Sin embargo, los productos de los raleos presentaron similares porcentajes de madera destinada a aserrado (15\%), a postes y varas (30\%) y leña (55 \%) (Peri et al., 2005b).

Por su parte, Martínez Pastur et al. (2008) determinaron el potencial de cosecha y el rendimiento industrial de ñirantales de Tierra del Fuego en rodales cuya área basal original era de $59 \mathrm{~m}^{2} / \mathrm{ha}$ con una intensidad de raleo que dejó un remanente de $30 \mathrm{~m}^{2} /$ ha. El volumen cosechado fue de $102 \mathrm{~m}^{3} / \mathrm{ha}$, y el rendimiento en aserradero varió con la calidad y el tamaño de las trozas desde $34 \%$ para trozas $>30 \mathrm{~cm}$ de diámetro en punta fina de calidad alta (pudrición blanca $<10 \%$ en la peor cara, pudrición parda $<30 \%$, mancha 
$<50 \%$, flecha $<3 \mathrm{~cm} / \mathrm{m}$, rajaduras $<50 \mathrm{~cm}$ y $\sin$ fustes retorcidos) a $4 \%$ para trozas de baja calidad. Las trozas de mejor calidad produjeron $9 \%$ de tablones, $21 \%$ de tablas, 49 $\%$ de tirantes, $5 \%$ de madera corta y $16 \%$ de madera para pallet. Los resultados sugieren la posibilidad de incorporar al aserrado sólo trozas de alta calidad de cualquier diámetro, lo que representa rendimientos de cosecha de $50 \mathrm{~m}^{3} / \mathrm{ha}$ para bosques de calidad de sitio media-alta. La inclusión de madera para pallets (producto que usualmente no se produce en el aserradero) incrementó significativamente el rendimiento en el aserradero.

En el manejo silvícola, la continuidad del estrato arbóreo del bosque nativo de ñire bajo uso silvopastoril tiene el objetivo de mantener la productividad y los servicios ambientales del sistema en el tiempo. Trabajos previos (Tejera et al., 2005; Peri et al., 2006a; Hansen et al., 2008) concluyen que la continuidad del estrato arbóreo bajo uso silvopastoril no puede asegurarse a través de la regeneración por semillas, por lo que fue necesario generar técnicas silviculturales que mantengan la sustentabilidad del sistema. En este sentido, una propuesta es la instalación de clausuras temporales de la regeneración pre-establecida por cepa, de semilla o raíz (o en sitios que no existiera regeneración, la forestación con plántulas de ñire obtenidas de vivero) que protejan las plantas del pastoreo y el ramoneo hasta que las mismas adquieran una altura superior a 2,5 m. Se estimó que se deberá protegerse de 2 a 5 renovales de ñire por ha/año hasta asegurar el reemplazo total de los individuos en fases de envejecimiento o con edades superiores hasta lograr la densidad final definida en cada mancha de bosque homogéneo (Peri et al., 2009b). En el caso de los ñirantales que se encuentran en la zona de ecotono con la estepa (ñirantales creciendo en condiciones de estrés hídrico severo) se deberían proteger hasta 250 árboles/ha, mientras que en ñirantales creciendo en zona más húmedas (por ejemplo cerca del bosque de lenga) se debería proteger un mínimo de 150 árboles/ha.

\section{PRODUCCIÓN Y CALIDAD FORRAJERA DEL SOTOBOSQUE}

La producción del sotobosque en sistemas silvopastoriles de ñire en la región patagónica sur tiene relación directa con la cobertura del dosel arbóreo y con la temperatura y régimen hídrico interactuando con los diferentes niveles de sombra. Por ejemplo, en la zona de El Foyel (Río Negro) se evaluó la producción del sotobosque de ñire en tres densidades de cobertura arbórea (Somlo et al., 1997). Los resultados indican que en un bosque ralo (300 a 500 árboles/ha) la producción de gramíneas fue mayor que en bosques de mayor densidad, con una producción media fluctuando desde 1.129 hasta $2.909 \mathrm{Kg} \mathrm{MS} / \mathrm{ha}$. Para la misma zona, Sarasola et al. (2008a) determinaron para dos años de evaluación (2006-2007) que la productividad media forrajera del sotobosque varió desde $1.106 \mathrm{Kg} \mathrm{MS} / \mathrm{ha}$ para un bosque de ñire denso (60\% de cobertura) con suelos subhúmedos hasta $2.575 \mathrm{Kg} \mathrm{MS/ha} \mathrm{para} \mathrm{un} \mathrm{ñirantal} \mathrm{ralo} \mathrm{(30} \mathrm{\%} \mathrm{cobertura)} \mathrm{en} \mathrm{sitios}$ de suelos húmedos.

En Chubut, se evaluó la productividad forrajera y la composición por grupos de especies (gramíneas, leguminosas y otras especies) en bosques de ñire desarrollándose en distintas condiciones de sitio (húmedos correspondientes a bosques de $15 \mathrm{~m}$ de altura y secos correspondientes a ñirantales de 3-4 m de altura) y en distintas condiciones de cobertura arbórea (Fertig et al., 2007). Los sitios húmedos presentaron una disponibilidad 
media 2,5 veces mayor que los sitios secos (1.288 vs $565 \mathrm{Kg} \mathrm{MS} / \mathrm{ha}$ ), y las situaciones entre copas presentaron una disponibilidad total promedio (1.217 Kg MS/ha) superior a las situaciones de cobertura de copas densas (418 Kg MS/ha). Además, Fertig et al. (2009) determinaron que la disponibilidad forrajera total, luego de cuatro años de realizados los raleos, se incrementó desde un $75 \%$ en los sitios secos (bosque bajo, $<4 \mathrm{~m}$ de altura, 50 $\%$ cobertura) hasta casi 5 veces en los sitios intermedios (bosque medio de 4 a $8 \mathrm{~m}$ y 40 $\%$ cobertura). En ambientes húmedos (bosque alto mayor a $8 \mathrm{~m}$ de altura), los autores detectaron que la producción del sotobosque aumentó desde $808 \mathrm{Kg} \mathrm{MS} /$ ha (bosque testigo sin ralear) a $2.002 \mathrm{Kg} \mathrm{MS} / \mathrm{ha}$ (50\% cobertura), determinado por un incremento principalmente en la disponibilidad de gramíneas y leguminosas.

En Patagonia Sur (Santa Cruz y Tierra del Fuego) en el límite entre estepa y bosque, el clima determina un régimen con un fuerte déficit hídrico coincidente con la estación de crecimiento. En estos sitios, el pastizal sometido a un sombreado y a la protección del efecto desecante de los fuertes vientos presenta menores tasas de transpiración y evaporación en comparación con sitios abiertos. Esta diferencia en la disponibilidad de agua en suelo en los sistemas silvopastoriles en comparación con pastizales de áreas sin árboles determinó una mayor productividad. Por ejemplo, en estos sitios de severo estrés hídrico se alcanzo la máxima producción de materia seca con una cobertura de copas del $55 \%$ (Figura $N^{\circ} 1$ ). En contraste, con un régimen de precipitaciones más favorable, se detectó una disminución de la tasa de crecimiento de materia seca de la pastura aproximadamente lineal con el aumento de la cobertura de copas desde $23,3 \mathrm{Kg}$ MS/ha/día en pastizales creciendo en la zona adyacente sin árboles a 4,2 Kg MS/ha/día con un $95 \%$ de cobertura de copas. Sin embargo, la presencia de árboles en estos sitios disminuyó el daño directo ocasionado por las heladas y/o acumulación de nieve sobre el pastizal. Por ello, los períodos vegetativos de los pastos se alargan en los sistemas silvopastoriles comparados a los de un pastizal abierto, modificando de esta manera el tiempo de oferta forrajera para los animales. Esta respuesta diferencial en la producción de materia seca de acuerdo a los diferentes niveles de sombra y estrés hídrico brinda una herramienta de criterio para determinar la intensidad de raleo, pudiendo ser mas intenso en sitios con moderado o sin estrés hídrico. Para acompañar el entendimiento de la respuesta de producción de materia seca del sotobosque, se evaluó las variaciones microclimáticas (temperaturas del aire y suelo, humedad relativa del aire, velocidad de viento y precipitaciones) en bosques de ñire bajo uso silvopastoril desarrollándose en dos clases de sitio, comparados con áreas sin cobertura arbórea (Bahamonde et al., 2009a). 


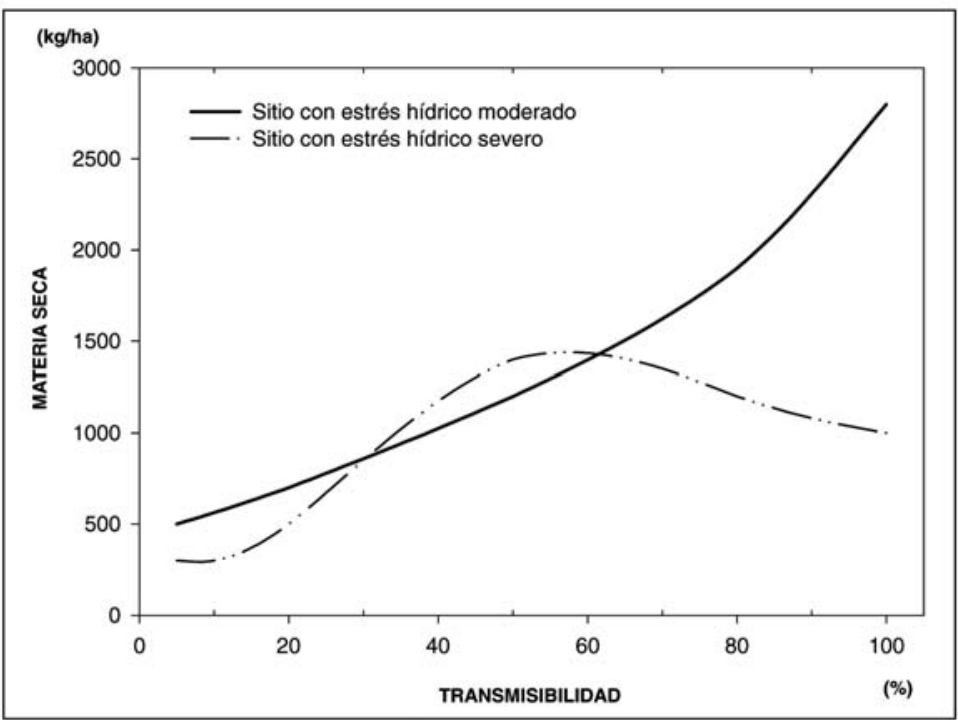

(Fuente: Peri, 2005)

Sitio con estrés hídrico severo ( $($ ) se correspondió con una humedad media del suelo hasta los $25 \mathrm{~cm}$ de profundidad inferior a $16 \%$ durante el principal periodo de crecimiento (Octubre-Abril) Sitio con estrés hídrico moderado (•) con una humedad media del suelo superior a $19 \%$.

\section{Figura $\mathbf{N}^{\circ} 1$ \\ PRODUCCIÓN MEDIA DE MATERIA SECA (MS) DEL PASTIZAL DESARROLLÁNDOSE EN SITIOS DE ÑIRANTALES CON DISTINTOS GRADOS DE COBERTURA DE COPAS Y ZONAS ADYACENTES SIN ÁRBOLES $(100 \%$ TRANSMISIBILIDAD).}

Además, existen antecedentes de producción de materia seca del pastizal mejorado a través de la introducción de pasturas forrajeras de alto rendimiento en sistemas silvopastoriles de ñire con diferentes niveles de radiación (Peri et al., 2005b; Peri et al., 2009c). La magnitud de la mejora en la productividad del pastizal con pasturas de trébol blanco (Trifollium repens) y pasto ovillo (Dactylis glomerata) estuvo en función del grado de sombreamiento. Por ejemplo, mientras que en sitios adyacente sin árboles (100 \% transmisibilidad luminosa) el aumento de producción de biomasa con la introducción de especies forrajeras representó un $35 \%$ respecto al pastizal natural, en el sistema silvopastoril con un $30 \%$ de transmisibilidad luminosa dicho aumento fue del $20 \%$ (Peri et al., 2005b).

Por su parte, en la zona de Río Turbio (51ㅜ 33' 10” LS, 7207' 35” LO) se instalaron jaulas de clausura en dos situaciones de transmisibilidad luminosa en un sistema silvopastoril de ñire: $20 \%$ (bajo copas) y $70 \%$ (entre copas), y en un pastizal puro sin árboles (100\% transmisibilidad), para determinar la respuesta productiva, composición botánica y calidad del pastizal con predominancia de trébol blanco. La productividad total 
de materia seca (MS) varió con la intensidad de luz siendo mayor en el sistema silvopastoril con $70 \%$ de transmisibilidad luminosa (Cuadro $\mathrm{N}^{\circ} 1$ ) relacionado con un mayor contenido de humedad del suelo (valor medio de $33 \%$ ). Sin embargo, la proporción media de trébol fue superior (49\%) en el pastizal sin árboles e inferior (35\%) en el pastizal creciendo bajo una transmisibilidad luminosa del $20 \%$ (Cuadro $N^{\circ} 1$ ). Estos resultados confirman la adaptación del trébol blanco a los sistemas con ñire en sitios húmedos, mejorando la calidad del pastizal natural.

\section{Cuadro $\mathrm{N}^{\circ} 1$ \\ PRODUCCIÓN TOTAL Y POR COMPONENTES DE MATERIA SECA DEL PASTIZAL CON TRÉBOL BLANCO (Trifollium repens)}

\begin{tabular}{|l|c|c|c|c|c|}
\hline Tratamiento & $\begin{array}{c}\text { Trébol } \\
\text { (kg MS/ha) }\end{array}$ & $\begin{array}{c}\text { Latifoliadas } \\
\text { (kg MS/ha) }\end{array}$ & $\begin{array}{c}\text { Gramíneas } \\
\text { (kg MS/ha) }\end{array}$ & $\begin{array}{c}\text { Material } \\
\text { Senescente } \\
\text { (kg MS/ha) }\end{array}$ & $\begin{array}{c}\text { Producción } \\
\text { Total } \\
\text { (kg MS/ha) }\end{array}$ \\
\hline $\begin{array}{l}100 \% \text { transmisividad } \\
\text { (pastizal sin árboles) }\end{array}$ & $1.120 \mathrm{a}$ & $320 \mathrm{a}$ & $490 \mathrm{ab}$ & $370 \mathrm{ab}$ & $2.300 \mathrm{a}$ \\
\hline $\begin{array}{l}\text { SSP 70\% } \\
\text { (entre copas) }\end{array}$ & $1.270 \mathrm{a}$ & $970 \mathrm{~b}$ & $700 \mathrm{a}$ & $670 \mathrm{~b}$ & $3.610 \mathrm{~b}$ \\
\hline $\begin{array}{l}\text { SSP 20\% } \\
\text { (bajo copas) }\end{array}$ & $720 \mathrm{~b}$ & $410 \mathrm{a}$ & $200 \mathrm{~b}$ & $230 \mathrm{a}$ & $1.560 \mathrm{c}$ \\
\hline
\end{tabular}

Letras diferentes indican diferencias significativas entre clones (Tukey, $p<0,05$ )

(Fuente: Peri et al., 2009b)

Patizal en el peak de biomasa (mes de Febrero 2008) correspondiente al período de crecimiento (Octubre 2007Mayo 2008) para un sitio adyacente sin árboles (100\% transmisibilidad luminosa) y para sistemas silvopastoriles en bosques de ñire (Nothofagus antarctica) con 20 (SSP $20 \%$ ) y 70\% de transmisibilidad (SSP $70 \%$ )

En forma similar a la productividad, Peri et al. (2005b) determinaron que la proteína bruta (PB) del pastizal de los ñirantales varió según la interacción entre los factores sitio e intensidad lumínica que ingresa al sotobosque, con un rango entre 8,2 y $12,2 \%$. En general, el contenido de PB fue mayor en los niveles de sombra severa (10\% de transmisibilidad) y en aquellos sitios de menor estrés hídrico. En contraste, la digestibilidad in vitro del pastizal (DIVMO) no presentó diferencias frente los diferentes niveles de sombra (Peri et al., 2005b). Mientras que los bajos valores anuales promedios de DIVMO $(55,6 \%)$ se detectaron en sitios o períodos de mayor déficit hídrico, la mejor digestibilidad media del pastizal (68,9\%) se observó en los sitios de menor estrés hídrico o en los períodos de primavera.

Fertig et al. (2009) determinaron que el contenido de PB en ñirantales del noroeste de la provincia de Chubut disminuyó en los sitios húmedos raleados (50 \% cobertura) respecto al bosque sin intervenir $(6,94$ vs $8,90 \%)$ debido probablemente a la dilución provocada por el crecimiento. Los mismos autores informaron que mientras la Fibra Detergente Neutro (FDN) aumentó en el pastizal de los sitios raleados (47,33 vs 40,7\%) lo cual podría provocar una disminución en el consumo potencial del ganado, los valores de Fibra Detergente Acido (FDA) no presentaron diferencias significativas (promedio 37,6 $\%)$. 
Para garantizar el uso silvopastoril de los ñirantales a nivel predial es necesario incorporar la evaluación de pastizales dentro del Plan de Manejo, ya que provee información para optimizar la producción ganadera y evitar el deterioro del sistema por sobrepastoreo. La evaluación de pastizales dará lugar a la Planificación del Pastoreo, el cual consiste en determinar el número de animales (carga animal) y la época de uso de cada potrero.

Recientemente se ha logrado desarrollar un método de evaluación de pastizales (Ñirantal Sur- San Jorge) adaptado al ecosistema de ñirantales en Patagonia Sur (Santa Cruz y Tierra del Fuego), el cual sirve como herramienta técnica para estimar la capacidad de carga animal en sistemas silvopastoriles a nivel predial (Peri, 2009a,b). El mismo se basó en la estimación de la Producción Primaria Neta Anual Potencial (PPNAP) del pastizal para diferentes condiciones del ñirantal y momentos de uso (primavera o peak de biomasa, verano, otoño e invierno).

Este método es de fácil uso, ya que las únicas variables que deben tomarse a campo para estimar la PPNAP (presentadas en una tabla de triple entrada), son la cobertura de copas (CC), la clase de sitio expresado por la altura promedio de los árboles dominantes (CS) y la cantidad de residuos leñosos (R) (Peri, 2009a,b). Las PPNAP registradas en los ñirantales fluctuaron desde $85 \mathrm{~kg} \mathrm{MS} / \mathrm{ha} / \mathrm{año}$ (pastizal en un ñirantal de CS III, CC de 5-30 \% y R de 30-50 \% utilizado en invierno) a $2.200 \mathrm{~kg} \mathrm{MS/ha/año} \mathrm{(pastizal} \mathrm{en} \mathrm{un} \mathrm{ñirantal}$ de CS I, CC 5-30 \% y R de 5-10\% utilizado en primavera).

La validación independiente $(n=20)$ indicó que el método captó un $83 \%$ de la variación de la PPNAP registrada en los ñirantales de Patagonia Sur. Sin embargo, es necesario ampliar la validación del método incorporando otras provincias como Chubut y Río Negro para detectar potenciales limitaciones del método, como así también monitorear la respuesta del pastizal bajo pastoreo con el fin de determinar la necesidad de ajustar la metodología propuesta.

\section{PRODUCCIÓN ANIMAL}

Estimaciones de la producción animal en ñirantales de Chubut, arrojan valores de $14 \mathrm{Kg}$ de carne vacuna/ha/año, lo cual aparece como un piso potencialmente mejorable ante las condiciones agro-ecológicas del área (Fertig, 2006). Para los establecimientos ganaderos ovinos de Patagonia Sur, el promedio del porcentaje de señalada es del $75 \%$ y la producción media de lana por animal de 4,7 Kg/animal (Ormaechea et al., 2009).

La principal dieta en la que se basa la producción ganadera (ovinos y bovinos) pastoreando los bosques de ñire a lo largo del año son las gramíneas y graminoides (56$90 \%$ ) donde se destacan Poa pratensis, Festuca pallescens, Holcus lanatus y Carex sp. (Manacorda et al., 1996; Bonino, 2006).

Existen antecedentes de ensayos que cuantificaron la respuesta de ovinos (ovejas Corriedale de 4 años de edad) y bovinos (vaquillonas Polled Hereford de 14 meses de edad) frente a la variación de los atributos del pastizal para dos coberturas arbórea (40 y $60 \%$ y pastoreando hasta dos condiciones de residual del pastizal (óptimo y sub-óptimo) 
en sistemas silvopastoriles de ñire en Santa Cruz (Peri et al., 2006b; Peri, 2008).

En ambos estudios, se midió la ganancia de peso vivo (GPV) individual (g/animal/ día) y por hectárea ( $\mathrm{kg} / \mathrm{ha} / \mathrm{día}$ ) durante el mes de diciembre coincidente con el peak de biomasa del pastizal y en parcelas de 0,7 ha.

Si bien no hubo diferencias significativas en GPV diario individual entre diferentes coberturas del sistema silvopastoril, las GPV disminuyeron entre un 50\% para el caso de ovinos y $86 \%$ para bovinos (Figura $\mathrm{N}^{\circ} 2$ ) cuando los animales pastorearon hasta un residual sub-óptimo. La mayor disponibilidad de pasto en el sistema silvopastoril con 40 $\%$ de cobertura de copas permitió una carga animal mas alta que el potrero con $60 \%$ de cobertura, resultando en una GPV por hectárea significativamente mayor para animales pastoreando hasta un residual óptimo (29,9 vs $17,1 \mathrm{~kg} / \mathrm{ha} / \mathrm{día}$ para bovinos y $3,8 \mathrm{vs}$. 2,4 $\mathrm{kg} / \mathrm{ha}$ /día para los ovinos).

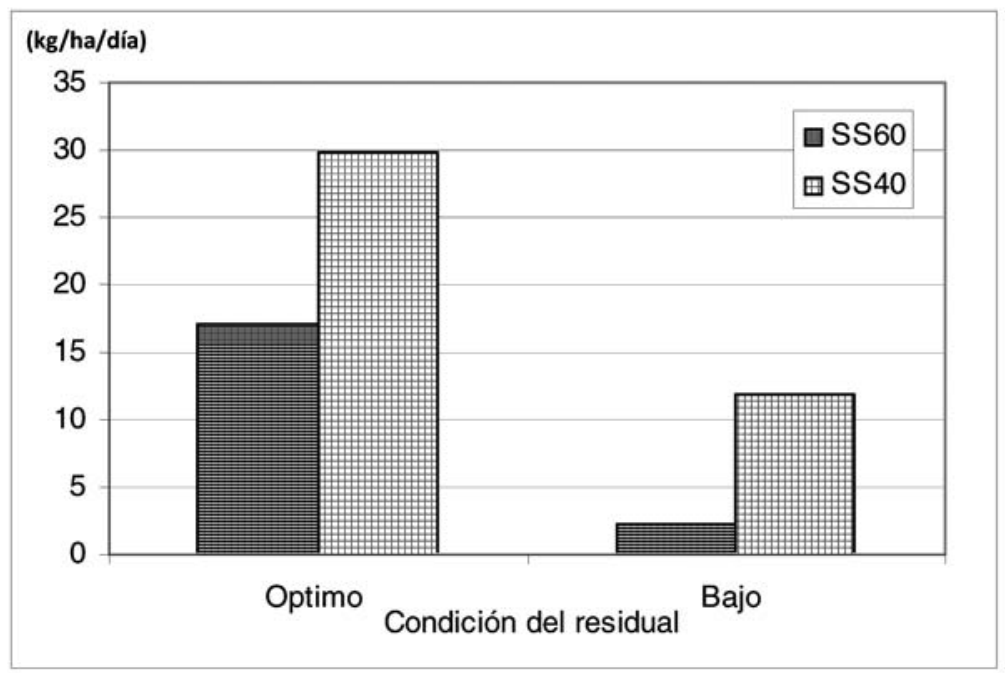

(Fuente: Peri, 2008)

(1). $40 \%$ (SS 40 ) y $60 \%$ (SS 60) de cobertura de copas con pastoreo para pastoreo con residual del pastizal óptimo y residual bajo, Patagonia Sur, Argentina. LSD 8,03 y 11,06 para residual del pastizal óptimo y residual bajo, respectivamente.

Figura $\mathrm{N}^{\circ} 2$

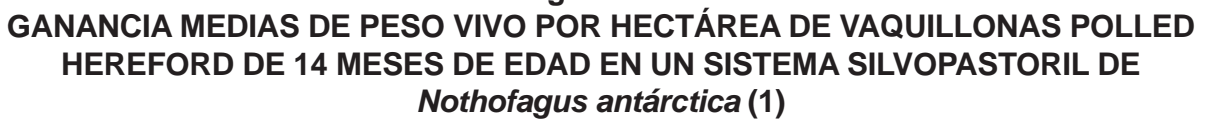




\section{MANEJO ANIMAL}

En Patagonia, existen diferentes sistemas de producción silvopastoril. Desde un punto de vista del manejo, Somlo et al. (1997) clasificaron a los sistemas silvopastoriles en:

(i) Sistemas nómadas principalmente en Neuquén donde ganado mixto de cabras, ovejas y vacas pastorean en la estepa en invierno y en bosque nativo durante el verano.

(ii) Sistema continuo anual de pastoreo en campos con bosques.

(iii) Pastoreo estacional con el uso de pastizales en tierras bajas con bosque nativo o el área ecotono-bosque en invierno y pastoreo en zonas altas con bosques en verano.

Según Laclau (1997), el componente para autoconsumo de la producción ganadera en Patagonia norte, enmarcado en sistemas silvopastoriles con rotaciones de veranadainvernada, es importante para la economía predominante de pequeños productores. Por su parte, el $78 \%$ de los establecimientos con ñire de Santa Cruz y Tierra del Fuego poseen un sistema de producción extensivo con bovinos y mixto (bovino+ovino), con valores de carga animal que fluctúan entre 0,60 y 0,65 ovejas/ha, y donde más del 75 $\%$ de las estancias presentan un manejo de los potreros en veranadas e invernadas (Ormaechea et al., 2009).

El manejo del pastoreo tiene una gran importancia en la producción ganadera. En Chubut se analizó los efectos de un sistema de pastoreo continuo (potrero de 10,1 ha) y un pastoreo rotativo (cuatro parcelas de 2,7 ha) durante 82 días en novillitos y vaquillonas Hereford de alrededor de 13-15 meses de edad en un ñirantal alto y abierto, con un pastizal conformado por especies de alto valor forrajero como Dactylis glomerata, Holcus lanatus, Trifolium pratense (trébol rojo) y Trifolium repens (trébol blanco) (Fertig, 2006). Si bien no hubo diferencias en la ganancia promedio de peso individual ( 1 kg/día/animal) entre sistemas, la producción de carne por unidad de superficie $(219 \mathrm{~kg} / \mathrm{ha})$ y la eficiencia de cosecha ( $57 \%$ ) bajo pastoreo rotativo fueron mayores que en el sistema continuo (174 $\mathrm{kg} / \mathrm{ha}$ y $40 \%)$.

Si bien se avanzó en la cuantificación de la producción del componente animal en estos sistemas silvopastoriles, los estudios fueron realizados en superficies pequeñas (potreros entre 0,7 y 10,1 ha) y en períodos cortos (20 a 82 días). Por lo tanto, fue necesario realizar estudios de producción y manejo animal a escala de establecimientos y que incorporen todo un ciclo productivo. En este sentido desde el año 2008 en la zona de Río Turbio (Santa Cruz) se iniciaros trabajos de investigación aplicada de sistemas de manejo animal a nivel de establecimiento y majada en ciclos productivos anuales. El objetivo de este trabajo fue comparar el Manejo Tradicional del Pastoreo (MTP) con una propuesta de Manejo Integral Silvopastoril (MIS), que incluyó la separación estratégica de ambientes (estepa, bosque y mallin) con el fin de aumentar los índices productivos de la ganadería ovina extensiva de la región (Ormaechea y Peri, 2011). 
Para el tratamiento MIS las divisiones de ambientes se realizaron mediante el uso de alambrado eléctrico de 4 hilos, con alturas de 90, 60, 40 y $20 \mathrm{~cm}$ buscando controlar también los corderos, y además se determinó la receptividad de los potreros según su disponibilidad inicial estimada a partir de evaluaciones de pastizales.

Para los animales del tratamiento MTP no se realizaron modificaciones de ningún tipo (la carga de los potreros fue determinada según los criterios del productor) y se respetaron los potreros tradicionales de veranada, invernada y parición, los cuales no se encuentran subdivididos en unidades ambientales. Cada tratamiento utilizó 1000 ovejas de la raza Corriedale de 6 dientes, debido a que es la que se encuentra en la etapa de mayor producción dado que ya ha tenido una parición y tiene su dentadura en condiciones adecuadas, pudiendo así mostrar los cambios en respuesta al manejo.

El comienzo del ensayo fue en el momento de señalada del establecimiento (febrero 2008) y los servicios fueron en el mes de Mayo luego de la esquila de ojo. La esquila general utilizada fue del tipo preparto (septiembre). Además, el grupo MIS pastoreó un potrero exclusivamente con bosque de ñire entre junio a septiembre, y utilizó solamente un mallín (sin carga durante la primavera) en enero.

Resultados preliminares demostraron que los potreros de MIS fueron utilizados con una carga acorde a su receptividad en todos los casos, mientras que el MTP registró una sobreutilización invernal (Ormaechea y Peri, 2011).

Se registraron valores levemente superiores de señalada y peso de vellones para el MIS (Cuadro $\mathrm{N}^{\circ} 2$ ), posiblemente producto de una carga moderada y del aprovechamiento del bosque y el mallín en momentos de mayor calidad del pastizal comparado con el resto de los ambientes.

Los valores superiores de condición corporal y ganancia de peso de ovejas en MTP, pudieron deberse a un menor número de hembras gestantes y por ende menores requerimientos nutricionales durante el período de octubre a enero.

\section{Cuadro $\mathrm{N}^{\circ} 2$ \\ VALORES MEDIOS PRODUCTIVOS DE MANEJO TRADICIONAL DEL PASTOREO (MTP) Y MANEJO INTEGRAL SILVOPASTORIL (MIS) EN UN ESTABLECIMIENTO DEL SUROESTE DE SANTA CRUZ (MEDIA \pm DESVÍO ESTÁNDAR)}

\begin{tabular}{|l|c|c|}
\hline & MIS & MTP \\
\hline Señalada (\%) & 93,3 & 90,4 \\
\hline Peso corderos (kg) & $31,2 \pm 5,20$ & $32,1 \pm 5,14$ \\
\hline Pérdida de peso invernal (kg) & 12,4 & 10,1 \\
\hline Ganancia de peso primaveral (kg) & 11,5 & 21,3 \\
\hline Condición corporal en último pesaje (rango 1-5) & $2,9 \pm 0.55$ & $3,2 \pm 0.46$ \\
\hline Peso de vellones (kg) & $4,64 \pm 0.83$ & $3,88 \pm 0.65$ \\
\hline
\end{tabular}

(Fuente: Ormaechea y Peri, 2011). 
En síntesis, la separación de ambientes homogéneos para su aprovechamiento racional parece tener resultados promisorios. Sin embargo, la falta de réplicas en los manejos evaluados, no permite extrapolar los resultados a todos los establecimientos de la región.

El valor de este tipo de estudio se fundamenta en la evaluación ecosistémica del sistema productivo, el cual contempló la utilización de potreros extensivos (300 a 5000 ha) a lo largo de una temporada anual de producción.

\section{BIOMASA Y CONTENIDO DE NUTRIENTES}

Existe un importante avance en el conocimiento de los sistemas silvopastoiles de ñire en lo que respecta a la dinámica y cambios en la compartimentalización aérea y subterránea de la biomasa y macro nutrientes (N, P, K, Ca, Mg y S) para gradientes de edad (desde fase de regeneración-5 años a fase de envejecimiento- 220 años), clases de copa (dominantes, codominantes, intermedios y suprimidos) y clases de sitio (Clase de Sitio $\mathrm{V}$ con altura de árboles dominantes menor a $6 \mathrm{~m}$ hasta Clase de Sitio III con alturas entre 8 y 10 m) (Peri et al., 2005c; Peri et al., 2006c; Gargaglione et al., 2008; Peri et al., 2008a; Gargaglione et al., 2009; Gargaglione et al., 2010). Mientras que la biomasa total acumulada varió, según la calidad de sitio y edad del rodal, desde 60,8 a 394,1 ton/ha, la acumulación total de nutrientes fluctuó entre 454 y 1.258 kg/ha.

A modo de ejemplo, en el Cuadro $N^{\circ} 3$ se presentan valores medios de acumulación de nutrientes en diferentes rodales de Nothofagus antarctica bajo uso silvopastoril en Patagonia, Argentina. Es importante resaltar que la proporción de biomasa y de la mayoría de los macro nutrientes del componente subterráneo fue superior al $50 \%$ en la fase de regeneración en todos los sitios estudiados. En sitios marginales, mientras que la concentración de nutrientes siguió el orden: hojas > corteza > raíces medias > ramas finas $>$ raíces finas $>$ albura $>$ raíces gruesas $>$ duramen, el orden de la acumulación de nutrientes en rodales maduros fue $\mathrm{Ca}>\mathrm{N}>\mathrm{K}>\mathrm{P}>\mathrm{Mg}>\mathrm{S}$ (Peri et al., 2008a).

Este tipo de información puede asistir a cuantificar el impacto de diferentes prácticas silvícolas y establecer pautas de manejo que tiendan a mantener la productividad del sistema silvopastoril en ñirantales a largo plazo. Por ejemplo, si el raleo forma parte del manejo silvícola, sería conveniente descortezar los fustes antes de retirarlos, evitando de esta manera la exportación fundamentalmente de Ca del sistema. Asimismo, al ralear y extraer árboles quedaría en el subsuelo las raíces que, al descomponerse, aportaría $\mathrm{P}$ que puede ser aprovechado por las pasturas. Con respecto al N, se podría hacer un raleo por lo bajo con una intensidad de extracción de hasta el $75 \%$, ya que el aporte de solo los árboles dominantes alcanza para cubrir los requerimientos del pastizal.

Sin embargo, numerosos factores afectan la disponibilidad efectiva de nutrientes al pastizal y al sistema en general, entre ellos, las tasas de descomposición, mineralización, el contenido de lignina, y otros.

Existen antecedentes donde se cuantificó el aporte anual, la distribución espacial y temporal de hojarasca y retorno potencial de nutrientes en bosques de ñire bajo manejo 
silvopastoril desarrollándose en diferentes clases de sitio (Peri et al., 2008b; Bahamonde et al., 2009b), e información sobre tasas de descomposición y mineralización en bosques con uso silvopastoril de Tierra del Fuego (Moretto et al., 2005; 2006; Bahamonde et al., 2009b). En este sentido, se determinó que la producción total de hojarasca en sistemas silvopastoriles de ñire fluctuó desde 967 a $2.117 \mathrm{~kg} \mathrm{MS} / \mathrm{ha}$ siendo mayor bajo copas (84 $\%$ del total) y durante el mes de abril (50 - $60 \%$ del total). Asimismo, el retorno potencial de nutrientes (principalmente $\mathrm{N}$ ) a través de la caída de hojarasca fue de $11,3 \mathrm{~kg} / \mathrm{ha}$ y la descomposición de hojas fue un $10 \%$ mayor en la posición entre copas (transmisibilidad de $66 \%)$.

La liberación de nutrientes de las hojas en descomposición varió en magnitud según el grado de luminosidad y los elementos evaluados. El $\mathrm{N}$ fue liberado de las hojas descomponiéndose bajo copas, mientras que entre copas fue inmovilizado. El P fue mineralizado en similares valores en las dos coberturas, el $\mathrm{K}$ fue inmovilizado en las dos situaciones de cobertura con distintas magnitudes.

Si bien rodales bajo uso silvopastoril disminuyen el aporte de hojarasca (principalmente por la disminución del número de árboles) y por ende su retorno potencial de nutrientes al suelo del bosque, esta disminución podría ser compensada en el corto plazo por una mayor descomposición de la materia orgánica en el lugar con mayor apertura de dosel o transmisibilidad. 


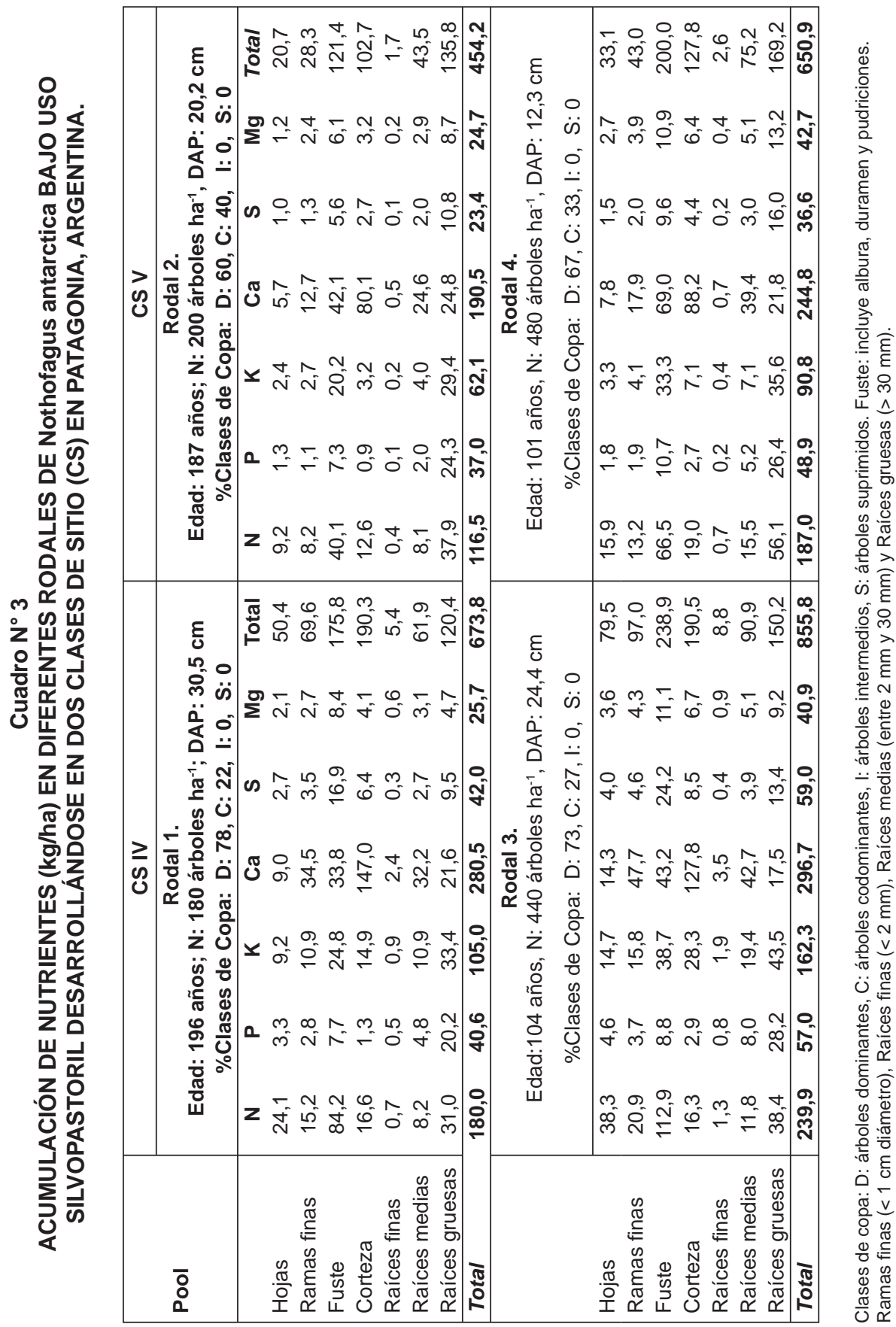




\section{FIJACIÓN DE CARBONO}

Actualmente son varios los estudios destinados a determinar la capacidad de secuestro de carbono (C) en los ecosistemas forestales, tanto en plantaciones con especies exóticas como en bosques nativos. Se ha demostrado que estos ecosistemas con un manejo adecuado pueden secuestrar mas carbono que otros ecosistemas terrestres (Dixon et al., 1994), contribuyendo notablemente a la mitigación del cambio climático producido por el constante incremento del $\mathrm{CO}_{2}$ atmosférico.

Para los sistemas silvopastoriles de ñire se cuantificó el contenido de carbono (C) del componente arbóreo basado en modelos de árboles individuales en diferentes fases de desarrollo (envejecimiento 111 - 220 años, crecimiento óptimo 21 - 110 años, regeneración 5 - 20 años), clases de copa (dominante, codominante, intermedio, suprimido) y clases de sitio (desde una altura media de árboles dominantes desde 5 a $12 \mathrm{~m}$ ) discriminado en sus principales compartimentos (hojas, ramas finas, albura, duramen, podredumbres, corteza, raíces finas y raíces gruesas).

Además se estimó la fijación de $C$ en el componente pastizal del sotobosques y la distribución de $C$ en el perfil del suelo hasta una profundidad de 0,6 m (Peri et al., 2005d; Peri et al., 2009d; Peri et al., 2010).

A modo de ejemplo, la acumulación de $C$ de un sistema silvopastoril desarrollándose en una Clase de Sitio IV (altura de los árboles dominantes de 7,8 m) con una densidad de 180 árboles/ha, de 196 años de edad y una proporción de clases de copa de $78 \%$ dominantes y $22 \%$ codominantes, fue de $252 \mathrm{t} / \mathrm{ha}$, de las cuales $11,9 \%$ fueron acumuladas en el estrato arbóreo, $1,4 \%$ en el pastizal y $86,7 \%$ en el suelo (Cuadro $\mathrm{N}^{\circ} 4$ ).

Es importante recalcar la importancia de evaluar la acumulación de $\mathrm{C}$ en los componentes subterráneos del estrato arbóreo (representó el 29,5\% del bosque) del sistema. 
Cuadro $\mathrm{N}^{\circ} 4$

\section{VALORES MEDIOS DE CARBONO (C) ACUMULADO EN DIFERENTES COMPARTIMENTOS DE UN SISTEMA SILVOPASTORIL EN PATAGONIA SUR, ARGENTINA.}

\begin{tabular}{|l|c|}
\hline COMPONENTE ARBOREO & (tC/ha) \\
\hline Hojas & 0,45 \\
\hline Ramas finas $(<1 \mathrm{~cm})$ & 1,03 \\
\hline Albura & 7,32 \\
\hline Duramen & 8,25 \\
\hline Corteza & 3,65 \\
\hline Podredumbre & 0,46 \\
\hline Raíces finas & 0,14 \\
\hline Raíces gruesas & 8,74 \\
\hline Total componente arbóreo & 30,1 \\
\hline COMPONENTE PASTIZAL & \\
\hline Hojas & 0,44 \\
\hline Muerto & 0,11 \\
\hline Macollo & 0,36 \\
\hline Raíces & 2,58 \\
\hline Total componente pastizal & 3,5 \\
\hline COMPONENTE SUELO & \\
\hline Mantillo (1 cm espesor) & 7,4 \\
\hline Horizonte orgánico (0-3 cm) & 13,6 \\
\hline Horizonte 1 mineral (3-10 cm) & 17,6 \\
\hline Horizonte 2 mineral (10-30 cm) & 81,2 \\
\hline Horizonte 3 mineral (30-60 cm) & 98,6 \\
\hline Total componente suelo & 218,5 \\
\hline Total del sistema silvopastoril & 252,1 \\
\hline
\end{tabular}

\section{CONSERVACIÓN DEL ÑIRANTAL BAJO USO SILVOPASTORIL}

Los sistemas silvopastoriles en los ñirantales de Patagonia son sistemas productivos desarrollados en bosques nativos, por lo que es estratégico asegurar su sustentabilidad. El sistema de Criterios e Indicadores (C\&I) permite abordar la complejidad de los sistemas silvopastoriles en forma jerárquica y lógica, otorgando herramientas para el monitoreo y fijando pautas de manejo (Rusch et al. (2009a). En este contexto, es importante contemplar (i) diseños adecuados de densidad de caminos, (ii) áreas de protección para el mantenimiento de la biodiversidad y servicios ambientales del bosque nativo, y (iii) sistemas permanentes de monitoreo que brinden información para mitigar los posibles efectos negativos de las intervenciones en el ñirantal y así elaborar protocolos de manejo que faciliten su sustentabilidad.

Según Rusch et al. (2009b), dos aspectos principales son claves para la sustentabilidad de los sistemas silvopastoriles en ñirantales; el mantenimiento de la 
capacidad productiva de los componentes arbóreo y forrajero, y el mantenimiento o mejora del bienestar de los actores asociados al manejo. Sugerencias y detalles respectos a este tema se encuentran en Rusch et al. (2004), Gallo et al. (2004), Carabelli y Peri (2005), Rusch et al. (2009a,b).

Como orientación, se debe considerar y privilegiar el uso y habilitación de caminos existentes en los predios, excepto en situaciones en las que su utilización pudiera provocar o agravar procesos erosivos. Según lo citado por Rusch et al. (2004), la densidad de caminos debe ser preferentemente menor a $30 \mathrm{~m} / \mathrm{ha}$. El área alterada por la red de caminos no debe superar un $5 \%$ de la superficie manejada. Al trazar caminos en valles 0 cerca de ellos, se debe evitar localizarlos al pie de laderas inestables o áreas de mucha humedad. Las pendientes máximas no deben superar el $12 \%$, excepcionalmente podrán trazarse tramos no mayores a 50 metros de longitud con $15 \%$ de pendiente. El camino debe tener una pendiente transversal o bombeo del 3 al $5 \%$. Detalles de los elementos de diseño de los caminos y drenajes se presentan en Rusch et al. (2004).

Áreas de protección y biodiversidad: Dentro de los predios deben considerase áreas de conservación. Respecto a los cauces de ríos y arroyos se deberá dejar una zona de protección de 15 a 60 m (Rusch et al., 2004). Los márgenes de lagunas y cauces deberían mantener la vegetación arbórea o arbustiva original minimizando las aberturas para el consumo por parte de los animales.

Además, la propuesta de manejo podría incluir la formación de bosques coetáneos en etapas sucesivas (cada 20 años, por ejemplo) de manera de establecer, a una escala de predio, bosques disetáneos que permitan mantener en todo momento bosques maduros e individuos en desmoronamiento que favorezcan la biodiversidad del sistema. En este sentido, para el mantenimiento de aves insectívoras de tronco deberán dejarse individuos enfermos y muertos; y mayores a $40 \mathrm{~cm}$ de diámetro para el anidamiento de aves como el carpintero magallánico o la lechuza ñacurutú (Gallo et al., 2004; Rusch et al., 2004). Asimismo, áreas de mantenimiento de arbustos permitirán la conservación de numerosas especies de aves e insectos (Rusch et al., 2004).

Monitoreo de las intervenciones: Debido al largo plazo que caracteriza a los procesos que ocurren en los bosques, resulta imprescindible monitorear los efectos de las intervenciones como el impacto en la producción, biodiversidad y estabilidad del rodal respecto al viento. A mediano y largo plazo se necesita evaluar el efecto del raleo a través de la instalación de parcelas permanentes de muestreo. Estas mediciones no solo contribuirán con información para mitigar los posibles efectos negativos para el ñirantal, sino que permitirán elaborar protocolos de manejo que faciliten su sustentabilidad. Herramientas de este tipo son demandadas por los organismos provinciales o nacionales de fiscalización y control. Las variables a monitorear se podrían basar en un principio en los indicadores presentados por Rusch et al. (2004) a escala predial y por Carabelli y Peri (2005) a escala provincial.

La confirmación de C\&I locales para el uso silvopastoril del bosque de ñire, contribuirá a la definición de los estándares Nacionales necesarios para los procesos de ecocertificación a escala predial. Esto adquiere especial relevancia para las empresas y 
productores que deseen obtener mercados internacionales que requieren la certificación de buen manejo forestal y ganadero.

\section{PAUTAS DE MANEJO}

El uso sustentable de los bosques nativos toma relevancia a partir de la promulgación de la Ley de Presupuestos Mínimos Ambientales para la protección de los bosques nativos, la cual podría financiar parte de los costos del manejo. Actualmente no se registran prácticamente Planes de Manejo presentados en las Direcciones de Bosques provinciales para el uso de los bosques de ñire en el marco del uso silvopastoril, presentándose además un insuficiente marco legal vigente en las provincias patagónicas que contemple el uso ganadero en estas masas forestales (Picco y Escalona, 2008).

Un manejo forestal sustentable debe abordar tres aspectos fundamentales; ser ecológicamente viable, económicamente factible y socialmente deseable. En este sentido, un rol importante de los organismos estatales sería reglamentar el uso de los bosques de ñire a través de planes de manejo. El uso silvopastoril en Patagonia Sur tendría que incorporar Planes de Manejo de largo plazo que tiendan a mantener en el tiempo los niveles de uso del bosque nativo de ñire ajustándose a las leyes forestales provinciales y nacionales, y que sirva como herramienta de los organismos de fiscalización y control. Para que se incorpore este mecanismo para el uso sustentable de los bosques nativos de ñire en Direcciones de Bosques provinciales, o como herramienta para el asesoramiento de los extensionistas al sector productivo, será necesario cumplimentar con los requisitos que se requieren para el manejo de las demás especies forestales nativas. En este contexto, fueron propuestas pautas generales para el manejo silvopastoril de los bosques nativos de ñire en Patagonia que tiendan a maximizar la producción del sistema y propender a su conservación (Peri et al., 2009a).

Los productores deberían cumplimentar las siguientes etapas a lo largo del sistema de producción:

(a) Realizar un inventario forestal para determinar las variables biométricas y geográficas de los bosques de ñire que se usarán como sistema silvopastoril. Estas deberán incluir la densidad y estado de la regeneración.

(b) Con los resultados del inventario forestal, la información productiva de la empresa y los objetivos del manejo del recurso forestal bajo estudio, se elabora un Plan de Manejo que incluye la información de base (estado legal, forestal y económico) y la planificación de las actividades silvícolas (raleos y/o protección de la regeneración) y de aprovechamiento para un período variable (se recomienda 5 años).

(c) En el caso de ser necesario de deben solicitar los permisos correspondientes para la construcción de los caminos de acceso.

(d) Una vez definidos las áreas de corta (área anual a intervenir), se deben realizar la marcación, la ubicación de las vías de saca y los canchones de acopio. 
(e) Una vez realizado la corta, se realiza la extracción de los productos madereros a los canchones de acopio. La mayoría de las veces los productos madereros deben ser fiscalizados antes de ser extraídos de los canchones de acopio.

(f) Para garantizar el uso silvopastoril de los ñirantales en el marco de la sustentabilidad también es necesario que el Plan de Manejo incorpore una Evaluación de Pastizales.

La Evaluación de Pastizales (utilizando por ejemplo el Método San JorgeÑirantal Sur previamente señalado) previa y posterior a las intervenciones silvícolas tendrá el objetivo de proveer información que permita tomar decisiones de manejo para optimizar la producción ganadera en los sistemas silvopastoriles.

La Evaluación de Pastizales dará lugar al la Planificación del Pastoreo, el cual consiste en determinar el número de animales (carga animal) y la época de uso de cada potrero. Se recomienda una dimensión de potrero no mayor a 1000 ha. Las etapas de la Planificación del Pastoreo son:

(1) Estimación de la receptividad de los potreros.

(2) Asignación de tipo de animal por potrero priorizando según situación, objetivos y requerimientos.

(3) Definición del sistema de pastoreo analizando las ventajas de pastoreo continúo versus pastoreo rotativo.

(4) Definición de número de cabezas según receptividad, categoría animal y época de uso. Cada 2 años será necesario realizar un monitoreo del pastizal para evaluar la respuesta del pastizal a los raleos.

(g) Dentro de los predios deben considerase las áreas de conservación del ñirantal como se señalara previamente.

\section{CONCLUSIONES Y LINEAMIENTOS FUTUROS}

Los resultados de las investigaciones y experiencias productivas posicionan a los sistemas silvopastoriles en bosques nativos de ñire como una alternativa productiva en varias regiones de Patagonia.

Se ha avanzado en el conocimiento de estos sistemas principalmente en la producción y calidad del componente forrajero e interacciones con el estrato arbóreo, y en menor medida en los aspectos relacionados a la producción animal. Además se destacan los avances en los estudios de ecofisiología y pautas para la conservación del ñirantal bajo uso silvopastoril.

El conocimiento de estas interrelaciones entre árboles-pastos-animales brinda 
actualmente herramientas para el manejo del sistema silvopastoril, las cuales optimizarán la producción y la sustentabilidad del recurso. Sin embargo, por tratarse de sistemas complejos, aún resta profundizar varios aspectos relacionados al manejo de estos sistemas silvopastoriles en Patagonia.

En este sentido, como lineamientos futuros de acción para el sector de investigación, productores e instituciones dedicadas al desarrollo se sugiere:

Profundizar estudios relacionados a la producción y manejo animal a escala de establecimientos durante todo un ciclo productivo.

Fortalecer la factibilidad de instalación de industrias primarias o secundarias alternativas (producción de tableros, parquet, muebles) con el fin de aumentar el valor agregado de los productos madereros provenientes de los sistemas silvopastoriles.

Seguir profundizando estudios de ecofisiología ya que permitirán entender los procesos e interacciones de estos sistemas como apoyo para un mejor manejo.

Si bien existen antecedentes de estudios económicos en sistemas silvopastoriles con ñire (Claps et al., 2004), es importante brindar herramientas económicas actualizadas y a diferentes escalas (predial y provincial) en el marco del uso silvopastoril.

Además, son necesarios futuros estudios que profundicen sobre el impacto a nivel paisaje, la conectividad para la vida silvestre a escala regional, el mantenimiento de la biodiversidad y los servicios ambientales.

Por último, es imprescindible integrar el conocimiento generado con políticas de desarrollo local, provincial y nacional para la expansión y mejor uso de los sistemas silvopastoriles en Patagonia.

\section{REFERENCIAS}

Bahamonde, H.A., Peri, P.L., Martínez Pastur, G., Lencinas, M.V., 2009a. Variaciones microclimáticas en bosques primarios y bajo uso silvopastoril de Nothofagus antarctica en dos Clases de Sitio en Patagonia Sur. Actas Primer Congreso Nacional de Sistemas Silvopastoriles, pp. 289-296. Posadas, Misiones, Argentina.

Bahamonde, H.A., Peri, P.L., Moretto, A., Monelos, L., 2009b. Producción de hojarasca, descomposición y dinámica de liberación nutrientes de hojas en un sistema silvopastoril de Nothofagus antarctica (G. Forster) Oerst. en Patagonia Sur, Argentina. Proceedings XIII World Forestry Congress, FAO. Theme: 1. Forests and biodiversity, Subtheme: 1.2 Deforestation and forest fragmentation, Buenos Aires, 18-25 Octubre 2009.

Bonino, N., 2006. Interacción trófica entre el conejo silvestre y el ganado doméstico en el noroeste de la Patagonia Argentina. Ecología Austral 16: 135-142. 
Carabelli, E., Peri, P.L., 2005. Criterios e Indicadores de sustentabilidad (C\&I) para el Manejo Sustentable de los Bosques Nativos de Tierra del Fuego - Una herramienta metodológica para la determinación de los C\&I en Patagonia, 88 pp. Ediciones INTA, Buenos Aires. ISBN 987-521-178-8.

Claps, L, Martínez Pastur, G., Peri, P.L., Vukasovic, R., 2004. Proyecto de Factibilidad. Alternativas de Manejo Sustentable para el Manejo Forestal Integral de los bosques de Patagonia. Proyecto de Investigación Aplicada a los Recursos Forestales Nativos (PIARFON). Dirección de Bosques de la Secretaría de Ambiente y Desarrollo Sustentable de Nación (SAyDS). Proyecto BIRF 4085-AR. 48 pp.

Dixon, R.; Brown, S.; Houghton, R.; Solomon, A.; Trexler, M.C.; Wisniewsky, J., 1994. Carbon pools and flux of global forest ecosystems. Science 263: 185-190.

Fertig, M., 2006. Producción de carne bajo distintos sistemas de pastoreo en ñirantales del Noroeste del Chubut. Carpeta Técnica, Ganadería № 21, Junio 2006. EEA INTA Esquel.

Fertig, M., Hansen, N., Tejera, L., 2007. Producción forrajera en bosques de Nothofagus antarctica (ñire). Actas IV Congreso Nacional sobre Manejo de Pastizales Naturales, 19 pp. San Luis, Argentina.

Fertig, M., Hansen, N., Tejera, L., 2009. Productividad y calidad forrajera en raleos en bosques de ñire Nothofagus antarctica. Actas Primer Congreso Nacional de Sistemas Silvopastoriles. Posadas, Misiones, Argentina.

Gallo, E., Lencinas, M.V., Peri, P.L., 2004. Biodiversidad en ñirantales. Alternativas de Manejo Sustentable para el Manejo Forestal Integral de los bosques de Patagonia. Informe Final del Proyecto de Investigación Aplicada a los Recursos Forestales Nativos (PIARFON), Tomo II: 645-670. Dirección de Bosques de la Secretaría de Ambiente y Desarrollo Sustentable de Nación (SAyDS). Proyecto BIRF 4085-AR.

Gargaglione, V., Peri, P.L., Martínez Pastur, G., 2008. Estimación de la biomasa aérea y subterránea de Nothofagus antarctica para diferentes clases de copa y edades. Actas Segunda Reunión sobre Nothofagus en la Patagonia - EcoNothofagus 2008, pp. 131136. Esquel, Chubut.

Gargaglione, V., Peri, P.L., Martínez Pastur, G., Rubio, G., 2009. Contenido de N, P y $\mathrm{K}$ en rodales de Nothofagus antarctica bajo un sistema silvopastoril en Santa Cruz, Argentina. Actas Primer Congreso Nacional de Sistemas Silvopastoriles, pp. 104-109. Posadas, Misiones, Argentina.

Gargaglione, V., Peri, P.L., Rubio, G., 2010. Allometric relations for biomass partitioning of Nothofagus antarctica trees of different crown classes over a site quality gradient. Forest Ecology and Management 259: 1118-1126.

Hansen, N., Fertig, M., Escalona, M., Tejera, L., Opazo, W., 2008. Ramoneo en 
regeneración de ñire y disponibilidad forrajera. Actas de la Segunda Reunión sobre Nothofagus en la Patagonia - EcoNothofagus 2008. Esquel, Chubut, pp. 137-142.

Ivancich, H., Soler, E., Martínez Pastur, G., Peri, P.L., Bahamonde, H., 2009. Índice de densidad de rodal aplicado al manejo silvopastoril en bosques de ñire (Nothofagus antarctica) en Patagonia sur. Actas Primer Congreso Nacional de Sistemas Silvopastoriles, pp. 245-250. Posadas, Misiones, Argentina.

Ivancich, H., Martínez Pastur, G., Cellini, J.M., Lencinas, M.V., Peri, P.L., 2010. Stand growth model for Nothofagus antarctica primary forests. Actas VI Southern Connection Congress, Session Ecology, Bariloche, Río Negro, Argentina, 15-19 Febrero 2010.

Laclau, P., 1997. Los ecosistemas forestales y el hombre en el sur de chile y argentina. Boletín Técnico № 34. Fundación Vida Silvestre Argentina, pp. 120.

Manacorda, M., Somlo, R., Pelliza Sbriller, A, Willems, P., 1996. Dieta de ovinos y bovinos en la región de los bosques de ñire (Nothofagus antarctica) de Río Negro y Neuquén. Comunicación Técnica № 59, Serie Pastizales Naturales, Área Recursos Naturales, INTA EEA Bariloche.

Martínez Pastur, G., Cellini, J.M., Lencinas, M.V., Peri, P.L., 2008. Potencialidad de la cosecha y rendimiento industrial de bosques de Nothofagus antarctica en Tierra del Fuego (Argentina). Actas IV Congreso Chileno de Ciencias Forestales, 10 pp. Talca, Chile, 1-3 Octubre 2008.

Moretto, A., Escobar, J., Peri, P.L., Livraghi, E., 2005. Tasa de descomposición en bosques de Nothofagus antarctica con uso silvopastoril en Tierra del Fuego. Actas XXX Jornadas Argentinas de Botánica, pp. 104. Rosario, Santa Fe.

Moretto, A., Escobar, J., Peri, P.L., Taier, R., 2006. Producción, descomposición y mineralización en bosques de Nothofagus antarctica con uso silvopastoril en Tierra del Fuego. Actas XXII Reunión Argentina de Ecología (ASAE), pp. 270. Córdoba.

Ormaechea, S.G., Peri, P.L., Molina, R., Mayo, J.P., 2009. Situación y manejo actual del sector ganadero en establecimientos con bosque de ñire (Nothofagus antarctica) de Patagonia sur. Actas Primer Congreso Nacional Sistemas Silvopastoriles, pp. 385-393. Posadas, Misiones, Argentina.

Ormaechea, S.G., Peri, P.L., 2011. Comparación de dos tipos de manejo del pastoreo ovino en la zona de ecotono bosque-estepa en Patagonia Sur. Actas IX International Grassland Congress, Rosario, Santa Fe, Argentina.

Peri, P.L., 2005. Sistemas Silvopastoriles en Ñirantales. IDIA XXI Forestal. Año V. N ${ }^{\circ} 8$ pp. 255-259.

Peri, P.L., Martínez Pastur, G., Monelos, L., Allogia, M., Livraghi, E., Christiansen, R., Sturzenbaum, M.V., 2005a. Sistemas silvopastoriles en bosques nativos de ñire: una 
estrategia para el desarrollo sustentable en la Patagonia Sur. En: Dinámicas Mundiales, Integración Regional y Patrimonio en Espacios Periféricos (Eds. Zárate R. y Artesi L.), pp.251-259. Universidad Nacional de la Patagonia Austral, Río Gallegos.

Peri, P.L., Sturzenbaum, M.V., Monelos, L., Livraghi, E., Christiansen, R., Moretto, A., Mayo, J.P., 2005b. Productividad de sistemas silvopastoriles en bosques nativos de ñire (Nothofagus antarctica) de Patagonia Austral. Actas III Congreso Forestal Argentino y Latinoamericano. Corrientes, $10 \mathrm{pp}$.

Peri, P.L., Gargaglione, V., Martínez Pastur, G., 2005c. Cambios en la compartimentalización aérea y subterránea de los macro nutrientes en gradientes de edad y clases de copa. Actas III Congreso Forestal Argentino y Latinoamericano, Comisión Silvicultura Bosque Nativo, 10 pp., Corrientes.

Peri, P.L., Viola, M.; Martínez Pastur, G., 2005d. Estimación del contenido de carbono en bosques de ñire (Nothofagus antarctica) en Patagonia Sur. Actas III Congreso Forestal Argentino y Latinoamericano, Comisión Deforestación, 9 pp., Corrientes.

Peri, P.L., Monelos, H.L., Bahamonde, H.A., 2006a. Evaluación de la continuidad del estrato arbóreo en bosques nativos de Nothofagus antarctica bajo uso silvopastoril con ganado ovino en Patagonia Sur, Argentina. Actas IV Congreso Latinoamericano de Agroforestería para la Pecuaria Sostenible. Varadero, Cuba, 6 pp.

Peri, P.L., Sturzenbaum, M.V., Rivera, E.H., Milicevic, F., 2006b. Respuesta de bovinos en sistemas silvopastoriles de ñire (Nothofagus antarctica) en Patagonia Sur, Argentina. Actas IV Congreso Latinoamericano de Agroforestería para la Pecuaria Sostenible, 7 pp., Varadero, Cuba.

Peri, P.L., Gargaglione, V., Martínez Pastur, G., 2006c. Dynamics of above- and belowground biomass and nutrient accumulation in an age sequence of Nothofagus antarctica forest of Southern Patagonia. Forest Ecology and Management 233: 85-99.

Peri, P.L., 2008. Respuesta de ovinos a pastizales creciendo en diferentes cobertura de copas en sistemas silvopastoriles de ñire (Nothofagus antarctica) en Patagonia Sur, Argentina. Zootecnia Tropical 26(3): 363-366.

Peri, P.L., Gargaglione, V., Martínez Pastur, G., 2008a. Above- and belowground nutrients storage and biomass accumulation in marginal Nothofagus antarctica forests in Southern Patagonia. Forest Ecology and Management 255: 2502-2511.

Peri, P.L., Bahamonde, H., Monelos, L., Martínez Pastur, G., 2008b. Producción de hojarasca en bosques primarios y bajo manejo silvopastoril de Nothofagus antarctica en la provincia de Santa Cruz, Argentina. Actas Segunda Reunión sobre Nothofagus en la Patagonia - EcoNothofagus 2008, pp. 149-155. Esquel, Chubut.

Peri, P.L., 2009a. Evaluación de pastizales en bosques de Nothofagus antarctica Método Ñirantal Sur. Actas Primer Congreso Nacional Sistemas Silvopastoriles, pp. 335- 
342, Posadas, Misiones, Argentina.

Peri, P.L., 2009b. Método Ñirantal Sur -San Jorge: una herramienta para evaluar los pastizales naturales en bosques de ñire. Carpeta Técnica EEA INTA Santa Cruz, pp. 3338, Sección 9. Producción Animal. Edición EEA Santa Cruz.

Peri, P.L., Hansen, N., Rusch, V., Tejera, L., Monelos, L., Fertig, M., Bahamonde, H., Sarasola, M. 2009a. Pautas de manejo de sistemas silvopastoriles en bosques nativos de Nothofagus antarctica (ñire) ñire en Patagonia. Actas Primer Congreso Nacional de Sistemas Silvopastoriles, pp. 151-164. Posadas, Misiones, Argentina.

Peri, P.L., Ormaechea S., Huertas L. 2009b. Protección de renovales de ñire. Carpeta Técnica EEA INTA Santa Cruz, pp. 15-16, Sección 4. Producción Forestal. Edición EEA Santa Cruz.

Peri, P.L., Mayo J.P., Christiansen R., 2009c. Evaluación de la producción y calidad de trébol blanco en sistemas silvopastoriles en ñirantales de Patagonia. Actas $\mathrm{V}$ Congreso Nacional - II Congreso Mercosur - I Jornada Técnica de Productores Sobre Manejo de Pastizales Naturales, pp. 176-177, Corrientes, 13-14 Agosto 2009.

Peri, P.L., Martínez Pastur, G., Gargaglione, V., Lencinas, M.V., 2009d. Aboveground and belowground carbon sequestration in a silvopastoral system of Nothofagus antarctica forests in Southern Patagonia, Argentina. Proceedings XIII World Forestry Congress, FAO. Theme: 1. Forests and biodiversity, Subtheme: 1.2 Deforestation and forest fragmentation, Buenos Aires, 18-25 Octubre 2009.

Peri, P.L., Gargaglione, V., Martínez Pastur, G., Lencinas, M.V., 2010. Carbon accumulation along a stand development sequence of Nothofagus antarctica forests across a gradient in site quality in Southern Patagonia. Forest Ecology and Management 260: 229-237.

Picco, O., Escalona, M., 2008. Sistemas productivos de Nothofagus antarctica en la Patagonia Argentina; Análisis de la legislación vigente. Actas de la Segunda Reunión sobre Nothofagus en la Patagonia - EcoNothofagus 2008. Esquel, Chubut, pp. 205-212.

Quinteros, C.P., Hansen, N., Kutschker, A., 2008. Diferenciación de la vegetación del sotobosque en diferentes tipos de bosque de ñire (Nothofagus antarctica) bajo uso silvopastoril. Actas de la Segunda Reunión sobre Nothofagus en la Patagonia EcoNothofagus 2008. Esquel, Chubut, pp. 44.

Rusch, V., Roveta, R., Peralta, C., Márques, B., Vila, A., Sarasola, M., Todaro, C., Barrios, D., 2004. Indicadores de sustentabilidad en sistemas silvopastoriles. Alternativas de Manejo Sustentable para el Manejo Forestal Integral de los bosques de Patagonia. Informe Final del Proyecto de Investigación Aplicada a los Recursos Forestales Nativos (PIARFON), Tomo II: 681-797. Dirección de Bosques de la Secretaría de Ambiente y Desarrollo Sustentable de Nación (SAyDS). Proyecto BIRF 4085-AR. 
Rusch, V., Sarasola, M., Hansen, N., Roveta, R., 2009a. Criterios e Indicadores como Método para guiar la Sustentabilidad. a-Principios generales, y Criterios e Indicadores ambientales en sistemas silvopastoriles con ñire (Nothofagus antarctica). Actas Primer Congreso Nacional de Sistemas Silvopastoriles. Posadas, Misiones, Argentina.

Rusch, V., Sarasola, M., Hansen, N., Roseta, R., 2009b. Criterios e Indicadores como Método para guiar la Sustentabilidad. b-Aspectos productivos y socioeconómicos en sistemas silvopastoriles con ñire (Nothofagus antarctica). Actas Primer Congreso Nacional de Sistemas Silvopastoriles. Posadas, Misiones, Argentina.

Sarasola, M., López, D., Gaitán, J., Siffredi, G., 2008a. Productividad de sistemas silvopastoriles en bosques de ñire en la cuenca del río Foyel. Actas de la Segunda Reunión sobre Nothofagus en la Patagonia - EcoNothofagus 2008. Esquel, Chubut, pp. 156-164.

Sarasola, M., Fernández, M.E., Gyenge, J., Peyrou, C., 2008b. Respuesta de los ñire al raleo en la cuenca del río Foyel. Actas de la Segunda Reunión sobre Nothofagus en la Patagonia - EcoNothofagus 2008. Esquel, Chubut, pp. 47.

SAyDS, 2005. Primer Inventario Nacional de Bosques Nativos. Ministerio de Salud y Ambiente de la Nación - Secretaría de Ambiente y Desarrollo Sustentable, Buenos Aires, pp. 86.

Somlo, R., Bonvissuto, G., Schlichter, T., Laclau, P., Peri, P.L., Allogia, M., 1997. Silvopastoral use of Argentine Patagonian forest. En: Temperate Agroforestry System (Ed. Gordon A.M. and Newman S.M.), pp. 237-250. Editorial CAB International, Wallingford, UK.

Tejera, L., Hansen, N., Fertig, M., 2005. Efecto de la cobertura arbórea y del pastoreo vacuno sobre el establecimiento de la regeneración de Nothofagus antactica (G. Forst) Oerst. Actas III Congreso Forestal Argentino y Latinoamericano. Corrientes, 7 pp. 